\title{
Quantum Anti-Zeno Effect
}

\author{
M. Lewenstein ${ }^{1}$ and K. Rzążewski ${ }^{1,2}$ \\ ${ }^{1}$ Institut für Theoretische Physik, Universität Hannover, D-30167 Hannover, Germany \\ ${ }^{2}$ Center for Theoretical Physics and College of Science, Polska Akademia Nauk, 02-668 Warsaw, Poland
}

(February 25, 2018)

We demonstrate that near threshold decay processes may be accelerated by repeated measurements. Examples include near threshold photodetachment of an electron from a negative ion, and spontaneous emission in a cavity close to the cutoff frequency, or in a photon band gap material.

03.65. Bz

The interplay between quantum dynamics and quantum measuments continues to attract attention of physicists since the birth of quantum physics [1]. One of the classic examples of such an interplay is the, so called, quantum Zeno effect [2]. It consists in modifying the quantum evolution by the repeated measurments. The essence of the quantum Zeno effect is that repeated interrogation if the system is still in the inital state tends to quench the system in this state as the frequency of interrogations grows. The reason for that is that the quantum evolution generated by the hermitian Hamiltonian is time reversible, and hence the probability $p(t)$ of intial state occupancy behaves as $p(t) \simeq 1-(t / \tau)^{2}$ for short times $t$, with $\tau$ being the characteristic time scale. If we make $N$ interrogations within the time $T$, and if each of them gives positive answer, i.e. collapses the actual state onto the initial state, then $p(T) \simeq\left(1-(T / N \tau)^{2}\right)^{N} \rightarrow 0$ as $N \rightarrow \infty$. It is worth stressing, however, that for decay processes into broad band reservoirs, $\tau$ is typically of the order of the inverse of the reservoir width (i.e. $10^{-16} \mathrm{~s}$ for laser induced ionization of an atom). For this reason, the Zeno effect in decay processes is very hard to observe [3].

Following the idea of R. Cook [4], the quantum Zeno effect has been observed in a coherent process of Rabi oscillations in a two level system [5]. The idea of Zeno effect was also employed in recent experiments on interaction free measurements and imaging [6]. The need for the collapse of the wave packet in the quantum Zeno effect has been critically analyzed in Ref. [7].

In this Letter we demonstrate that under suitable conditions, the decay of some quantum systems from an initial state might be accelerated by frequent interrogations whether the system remains in that state. We call this phenomenon a quantum anti-Zeno effect. The effect takes place in systems that decay near threshold. In fact, we show that even below threshold, a frequent interruption of the evolution may enforce a complete decay of otherwise partially stable system. Somewhat similar effect has been proposed by Gontis and Kaulakys [8], who argued that frequent interrogations may destroy localization effect [9] in quantum chaotic systems, and may enforce classical stochastic motion.

Near threshold decay occurs in several physical situations. Here we discuss: a) near threshold photodetachment of an electron from a negative ion [10], b) spontaneous emission in a cavity close to cutoff frequency [1], or in a photon band gap medium [12]. The dynamics of all these systems is governed by the following generic Hamiltonian

$$
\begin{aligned}
H & =\hbar \Delta|0\rangle\left\langle 0\left|+\int \hbar \omega(k)\right| k\right\rangle\langle k| d k \\
& +\hbar \int(g(k)|0\rangle\langle k|+\text { h.c. }) d k,
\end{aligned}
$$

where $|0\rangle$ is the decaying state (ground state for ionization, excited state for spontaneous emission), and $k$ is a multi-index enumerating the states in the final continuum. In the case a) of photodetachement these are just the final states of an electron, in the case b) of spontaneous emission these are products of the atomic ground state and single photon states characterized by a wave vector and a polarization denoted by $k$. In Eq. (11) rotating wave approximation (RWA) has been used [13.

In the case a), the detuning $\Delta$ is the difference between the ionizing laser frequency and the ionization potential divided by $\hbar$. The label $k$ can be identified with $\omega(k) \equiv$ $\omega$, where $\hbar \omega$ is the energy of the photodetached electron. Various models of the coupling $g(\omega)$ have been discussed in the literature. The angular momentum $l$ of the final states determines the behaviour of $|g(\omega)|^{2} \propto \omega^{l+1 / 2}$ for small $\omega$ in accordance with the Wigner's law 14. In the following we will use the expression

$$
|g(\omega)|^{2}=\frac{A}{\pi} \frac{\sqrt{\beta \omega}}{\omega+\beta},
$$

for $\omega \geq 0$ in the $s$-continuum. In the above expression $\beta$ is the continuum width, and $A$, which is proportional to the laser intensity, is the Fermi Golden Rule photodetachment rate far above the threshold [10].

In the case b) of spontaneous emission $\Delta$ is the difference between the transition frequency and the cavity cutoff, or band gap edge frequency, $\omega_{c}$. The $\omega(k) \geq 0$ describes the dispersion relation; near the boundary of the gap in the isotropic model it is well approximated by

$$
\omega(k)=\frac{\omega_{c}}{k_{0}^{2}}\left(k-k_{0}\right)^{2}
$$


where $k_{0}$ is the wave vector at $\omega_{c}$. In the relevant region of $k \simeq k_{0}, g(k)$ varies slowly, and can be taken as a constant proportional to the atomic transition dipole moment $d$ 11, 12.

The survival amplitude in the initial state $|0\rangle, \alpha(t)$, maybe written as an inverse Laplace transform

$$
\alpha(t)=\int_{\Gamma} \frac{e^{z t}}{\mathcal{H}(z)} \frac{d z}{2 \pi i},
$$

where the countour $\Gamma$ runs parallely to the imaginary axis and is placed to the right from all singularities of the integrand, whereas the resolvent function $\mathcal{H}(z)$ is given by

$$
\mathcal{H}=z+i \Delta+\int \frac{|g(k)|^{2}}{z+i \omega(k)} d k
$$

In various regimes of parameters the following singularities contribute to the time evolution. Above the threshold, there exists a Wigner-Weisskopf pole with a negative real part. The contribution of this pole represents exponentially damped oscillating term. Both above and below the threshold there is a contribution from the cut in the complex plane. The cut may be taken along the negative part of the real axis. The cut contribution gives in particular the celebrated algebraic long tail of the evolution [15]. The threshold in the model does not correspond to $\Delta=0$, and instead is dynamically shifted by

$$
\Delta_{c}=\int \frac{|g(k)|^{2}}{\omega(k)} d k
$$

For the model a) the threshold is shifted to $\Delta_{c}=A$, whereas for the models b) it is pushed toward infinity. The latter effect is caused by the fact that the density of photon modes has a singularity for $\omega(k)=0$ [16. Below the dynamically shifted threshold, there exists a purely imaginary pole, which contributes a non-decaying, purely oscillatory term to $\alpha(t)$. Thus, sharp edge versions of the model b) exhibit always incomplete damping. Of course, all of the above discussed contributions sum up to $p(t)=|\alpha(t)|^{2} \simeq 1-t^{2} / \tau^{2}$ for ultra short times. It is the steep temporal behavior of the cut contribution for moderately short times which we are going to exploit for the quantum anti-Zeno effect. In fact, close to threshold the model a) enters the regime of asymptotic decay for such times, and decays generically as $1 / t^{3}$. The exception occurs at the exact threshold, where the decay undergoes a crossover to the $1 / t$ behavior and significant "critical" slowing down. In particular, it is easy to see that if at such time scales $p(t) \simeq(\tau / t)^{\nu}$, for $t>\tau$ with $\nu>0$, then after $N$ interrogations within time $T$, the survival probability $p(T) \simeq(N \tau / T)^{N \nu}$ decreases to values of the order of $\exp \left(-\nu e^{-1} T / \tau\right)$ as $N$ grows from 1 to $\simeq e^{-1} T / \tau$.

The analytic expressions for $\alpha(t)$ can be easily obtained, and in the case a) and the model of Eq. (2) read

$$
\alpha(t)=\sum_{i=1}^{3} \frac{y_{i}\left(y_{i}+\sqrt{\beta}\right)}{3 y_{i}^{2}+2 \sqrt{\beta} y_{i}+\Delta} e^{\left(i y_{i}^{2} t\right)} \operatorname{erfc}\left[\exp (i \pi / 4) \sqrt{t} y_{i}\right]
$$

where erfc(.) denotes the complementary error function of complex argument, and $y_{i}$ are the (complex in general) roots of

$$
y^{3}+\sqrt{\beta} y^{2}+\Delta y+\sqrt{\beta}(\Delta-A)=0 .
$$

The corresponding result for the model b) is obtained by setting $\beta \rightarrow 0, A \rightarrow \infty$ in such a way that $A \sqrt{\beta} \rightarrow \gamma^{3 / 2}$, where $\gamma=\left[\omega_{c}^{7 / 2} d^{2} / 2 \hbar c^{3}\right]^{2 / 3}$.

As in the Refs. [4, 17, we propose to interrogate the decaying system using a series of short pulses of light resonant with a dipole allowed transition between the initial state and some other bound state of the atom. We consider the case when the pulse is intense enough to generate a large number of fluorescence photons, and much longer than the characteristic time scale of the decay. In such a case sufficient decoherence between the initial state and the continuum is incurred to interrupt and reset the coherent evolution [7]. If $N$ interruptions are made within time $T$, the survival probability becomes $p(T) \simeq|\alpha(T / N)|^{N}$.

In Fig. 1 we have plotted the survival probability after $T=100 / A$ with various number of interruptions (indicated) as a function of detuning in the vicinity of the dynamically shifted threshold. The upper thick curve corresponds to the fully coherent, uninterrupted dynamics. For the model a) the following parameters were used: $A=10^{4} \mathrm{~Hz}$, and $\beta / A=10^{6}$. Observation time was taken equal $T=10^{-2}$ s. We consider interrogation pulses of the duration $10^{-6} \mathrm{~s}$, i.e. sufficient to produce a lot of fluorescence photons for a typical dipole transition. Such pulses are short enough to be considered as instantenous on a time scale of the decay $\left(10^{-2}\right)$; up to 1000 of such pulses well separated by $10^{-5} \mathrm{~s}$ can be used. Both below and above threshold the more frequent interruptions cause faster decay. The reason for that is explained in Fig. 2 , which presents the survival probabilty in a logarithmic scale at the exact dynamical threshold as a function of time for the cases without, and with 200 interruptions with the time $T=10^{5} / A$. In this case the coherent decay is particularly slow and occurs on the time scale of few $10^{5} / A$. Clearly, the coherent decay is non-exponential, and already for very short times can be well approximated by the algebraic $1 / t$ dependence, which has very large derivative for such times. Measurements set the system back to the initial state, and essentially press it to exploit this very steepy initial part of the time dependence. In effect, the decay becomes much faster and exponential in character, $p(n T / N) \simeq(\tau N / T)^{n \nu}$. Apart from this acceleration of the decay, the measuments destroy the stability of the decaying state below threshold. Even though, in such a case the coherent evolution leads to 
non-vanishing probability of survival as $t \rightarrow \infty$, however, the frequent interruptions lead to the total decay. One should stress, the advantage of working with the model a) is that the time scale can be here cotrolled, since $A$ is proportional to the ionizing laser intensity. Values between $10^{4}$ to $10^{8}$ are experimentally feasible. Being close to threshold requires laser stability within the $\mathrm{A}$ range (i.e. in the worst case in the $\mathrm{kHz}$ range). Observation times are reasonable, and "quantum jumps" measuremt scheme could be implemented without problems. All that means, that quantum anti-Zeno effect in near threshold decay is easier to observe experimentally than its ultra short time analog - the quantum Zeno effect.

In principle, the same effects as discussed for the model a) can be observed in the case b), but here the time scales are not so favorable. depending whether we consider spontaneous decay in the cavity, or in the photon band gap medium, the reasonable values of $\gamma$ lie between $10^{5} \mathrm{~Hz}$ (Rydberg atoms in a microwave cavity, or waveguide) and $10^{9} \mathrm{~Hz}$ (optical and infrared transitions in band gap media). In the model b) the characteristic time scale of the decay is typically $\simeq 10 / \gamma$. For Rydberg atoms the decay would take place on the scale of hundreds of microseconds, and could thus be monitored using "quantum jumps" technique, which requires the time scales discussed above in the context of model a). The decay of an impurity atom in the photon band gap medium in the infrared or optical regime of photon frequencies would then take few nanoseconds, and would be rather hard to be interrupted by "quantum jumps" to another level.

It is worth stressing, that independently of the value of $\Delta$, the model b) leads to a non-vanishing survival probability as $t \rightarrow \infty$. Moreover, this final survival probability depends very critically on the detuning, and decreases very strongly below the threshold. This is illustrated in Fig. 3 for $\Delta=-\gamma, 0, \gamma$, which give final survival probabilities $p_{f} \simeq .7,0.45$, and 0.15 , respectively. Imagine that we perform "quantum jump" measurments in such a way, so that the intervals between measurements are at least of the order of $100 \mu$ s, i.e. larger than the time scale of the evolution $\simeq 10 / \gamma$. This condition is obviously fulfilled for an inpurity atom in the photon band gap medium, and marginally fulfilled for Rydberg atoms in a cavity. On such a long time scale the evolution from Fig. 3 looks like an instantenous initial slip follow by a constant. Depending on the detuning chosen the survival probability monitored by "quantum jumps" will tend to zero rapidly with the number of interruptions $n$ as $p_{f}^{n}$. Frequent observing if the Schrödinger cat is alive, kills it faster. Such experiment requires "tuning" of atomic transition frequency, or the photon band gap edge within the range of $\gamma$, which can be for instance done using external static electromagnetic field and Zeeman, or Stark effect.

Summarizing, we have shown that near threshold decay can be exploited to observe the quantum anti-Zeno effect. Typical near threshold decay processes are charac- terized by ultra short period of quadratic decay, followed by a long phase of non-exponential decay, with a very large rate at the begining, gradually slowing down in the course of the dynamics. Monitoring if the decaying system remains in the initial state, shifts the system back to the fast initial phase of the non-exponential decay. In effect, more frequent measurement, cause faster decay. We discussed experimental feasibility of observing this effect for two models describing a) near threshold photodetachment of an electron from a negative ion, and spontaneous emission from an atom located in a cavity, or from an impurity located within a photon band gap medium. Our scheme is quite general, and can be used for any decaying system with several time scales. Frequent minitoring of such systems will tend to diminish the role of the long time time scales, and to blow up the role and influence of the shorter time scales. In particular, frequent monitoring leads to full decay of otherwise partially stable systems. There are implications of our result for quantum error correction schemes in quantum computing [18], which typically employ quantum Zeno effect. Using computing units with non-standard decay, such as the ones discussed above, might impose serious limitations on, or require developent of new error correcting strategies.

The idea of this paper was born in fruitful discussions with Anna Sanpera. We acknowledge the support of Deutsche Forschungsgemeinschaft (SFB 407). K.R. thanks the Humboldt Foundation for its gereous support.

FIG. 1. Survival probability for the model a) as a function of detuning after time $T=100 / A$. The thick upper curve corresponds to the coherent, uninterrupted evolution, whereas the numbers of interruptions for other curves are indicated.

FIG. 2. Survival probability for the model a) as a function of time exactly at the dynamically shifted threshold. The upper curve corresponds to the coherent, uninterrupted evolution, whereas the lower curves was obtained applying 50 (200) interruption pulses within the time $2 \times 10^{5} / A,\left(10^{5} / A\right)$ respectively.

FIG. 3. Survival probability for the model b) as the function of time. The values of $\Delta$ are indicated. 
[1] J. A. Wheeler and W. H. Żurek, Quantum Theory and Measurement (Princeton University Press, Princeton, 1983).

[2] B. Misra and E. C. G. Sudarshan, J. Math. Phys. 18, 756 (1977).

[3] J. Schwinger, Ann. Phys. 9, 169 (1960); L. Fonda et al., Rep. Prog. Phys. 41, 587 (1978).

[4] R. Cook, Phys. Scr. T21, 49 (1988).

[5] W. M. Itano et al., Phys. Rev. A41, 2295 (1990).

[6] P. G. Kwiat et al., Phys. Rev. Lett. 74, 4763 (1995); for theoretical foundation see A. C. Velitzur and L. Vaidman, Found. Phys. 23, 987 (1993), for recent references A. G. White et al., quant-ph 9803060 .

[7] V. Frerichs and A. Schenzle, Phys. Rev. A44, 1962 (1991).

[8] V. Gontys and B. Kaulakys, Lith. J. Phys. 38, 118 (1998); quant-ph/980615; Phys. Rev. A56, 1131 (1997).

[9] G. Casati et al., Phys. Rep. 154, 77 (1987); F. M. Izrailev, Phys. Rep. 196, 229 (1990).

[10] (a) K. Rzążewski et al., J. Phys. B15, L661 (1982); (b) S. L. Haan and J. Cooper, J. Phys. B17, 3481 (1984).

[11] M. Lewenstein et al., J. Phys. B21, L9 (1988); M. Lewenstein et al., Phys. Rev. A38, 1075 (1988).

[12] see for instance S.-Y. Zhu et al., Phys. Rev. lett. 79, 205 (1997); S. Bay et al., ibid., 2654 (1997); T. Quang et al., ibid, 5238 (1997) and references therein.

[13] To obtain an accurate value of the dynamical threshold shift $\Delta_{c}$ (see below), non-RWA corrections have to be considered [10].

[14] E. P. Wigner, Phys. Rev. 73, 1002 (1948).

[15] L. A. Khalfin, Sov. Phys. JETP 6, 1053 (1958).

[16] In realistic calculations, this singularity is always regularized, see for instance Ref. 11.

[17] Such detection employing quantum jumps to a side level in the context of near threshold decay of unstable states was discussed in Ref. [17], J. Grochmalicki and M. Lewenstein, Phys. Rev. A40, 2517 (1990).

[18] P. W. Shor, Phys. Rev. A52, R2493 (1995); A. M. Steane, Phys. Rev. Lett. 77, 793 (1996); Proc. Roy. Soc. Lond. A452, 2551 (1996). 


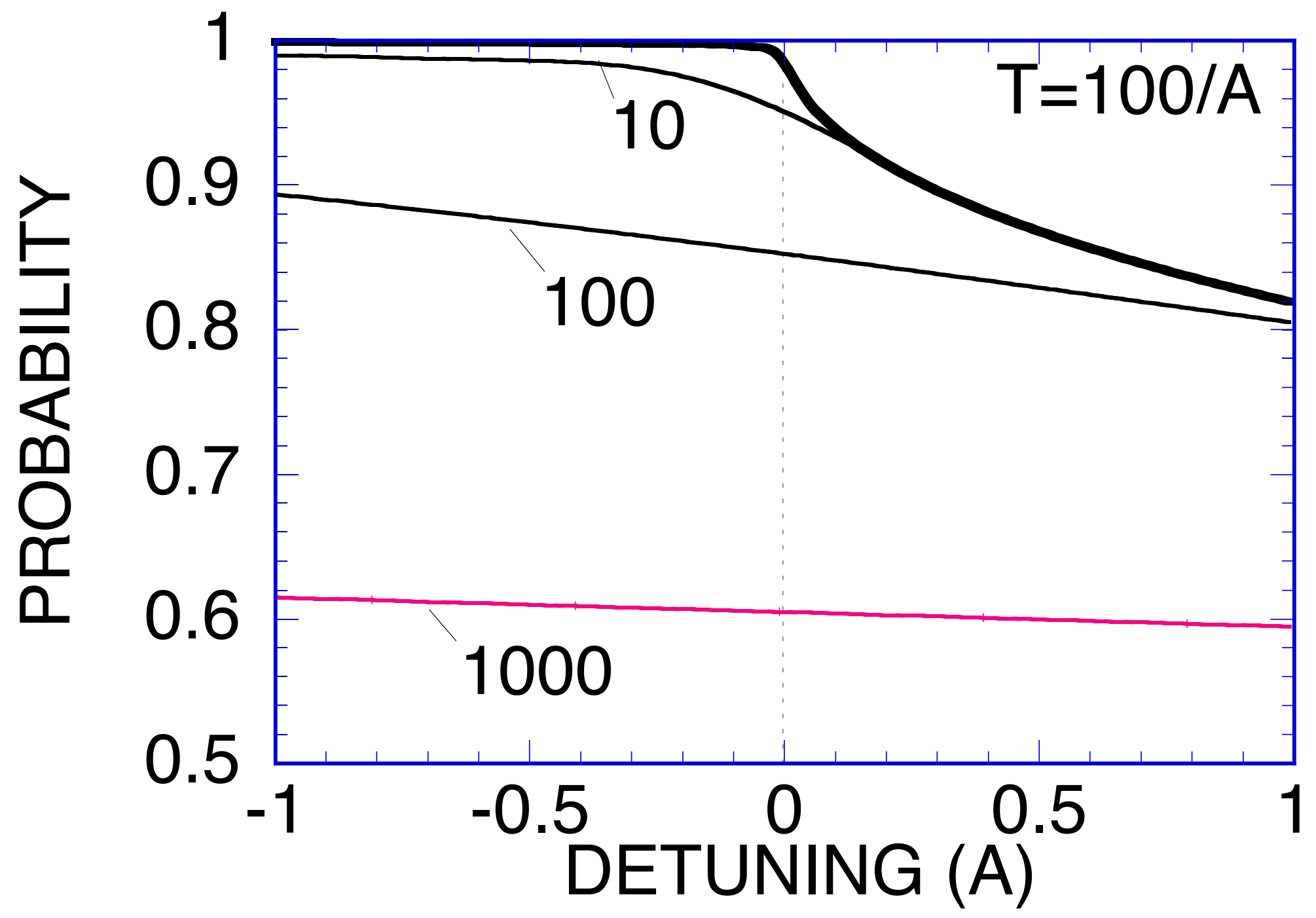




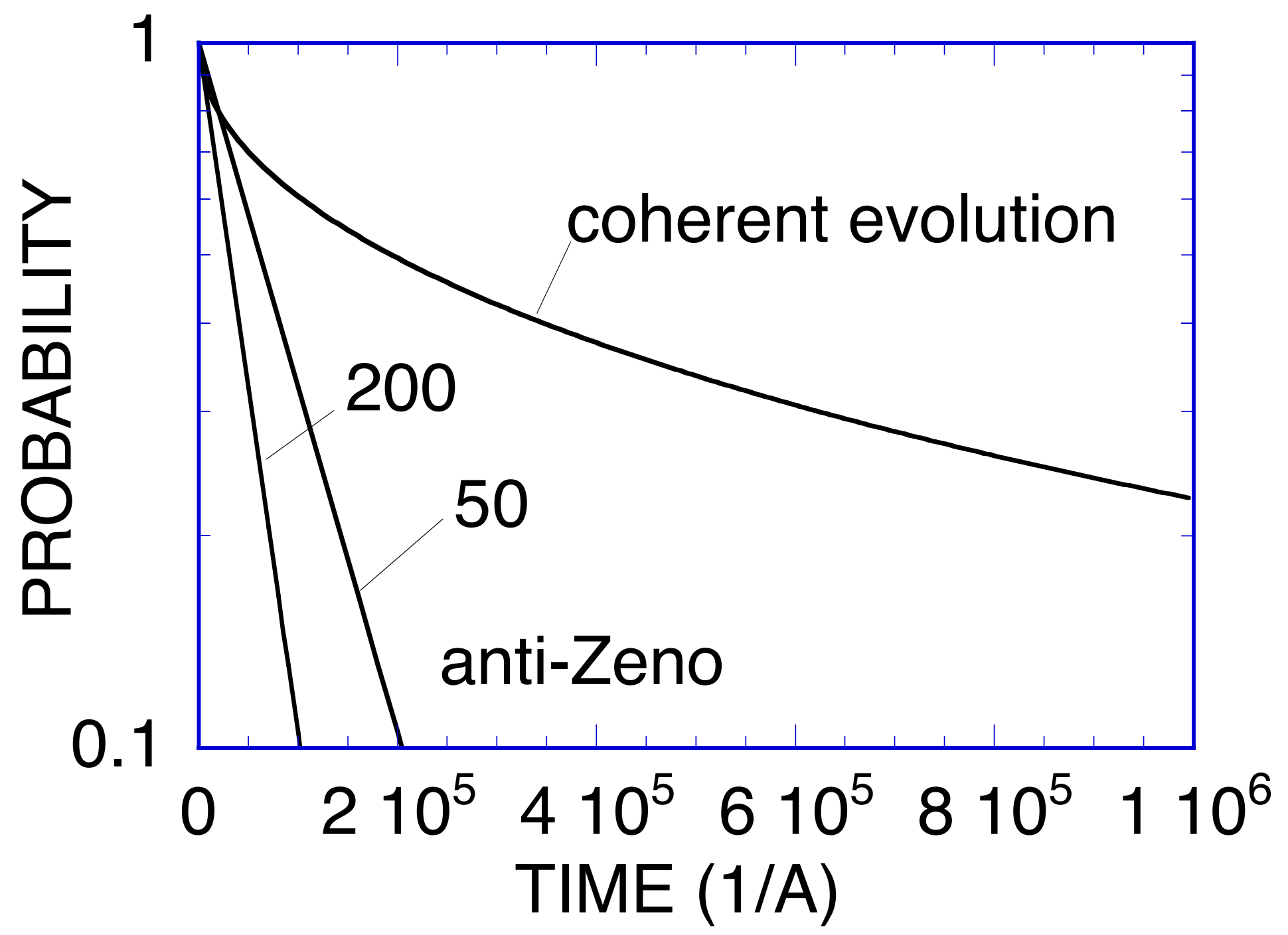




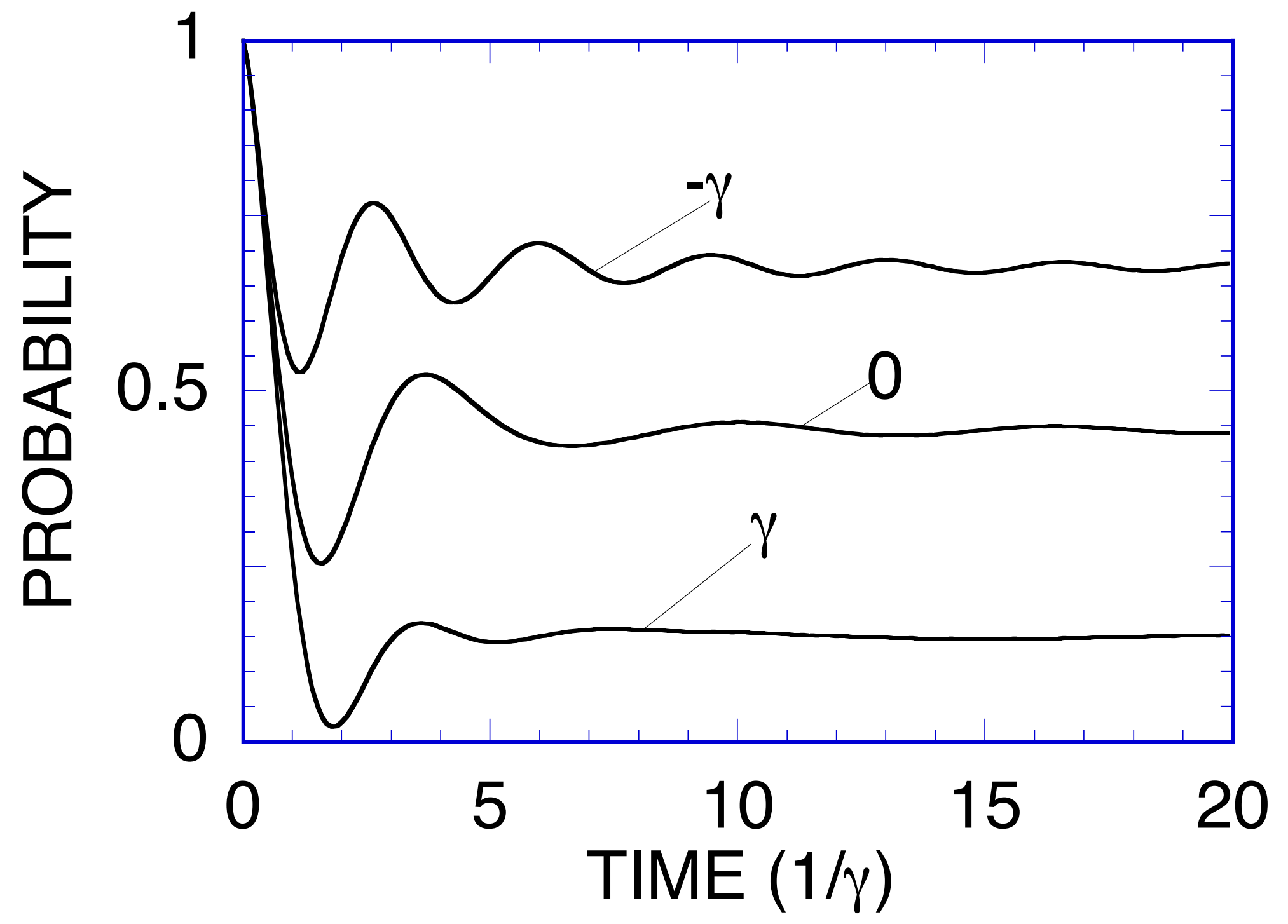

